\title{
MRI in the management of suspected cervical spondylotic myelopathy
}

\author{
P F Statham, D M Hadley, P Macpherson, R A Johnston, I Bone, G M Teasdale
}

\begin{abstract}
One hundred and two patients with suspected cervical spondylotic myelopathy were prospectively investigated using MRI as the initial imaging technique. The aim was to discover if clinicians could manage patients with MRI alone, or if they would find a second investigation necessary. Eighty two patients were managed using MRI alone, 34 of whom were treated surgically. Twenty patients had a second investigation: a myelogram in 18 and a CT myelogram in two. This was performed in nine patients to exclude structural pathology in the thoracic or lumbar region (which was not examined with MRI), and in 11 to obtain more specific information about the cervical region. Only five of these 20 patients had surgical treatment. The diagnosis changed after the second investigation in four patients, but management was not influenced in any of these. MRI is a satisfactory alternative to myelography for most patients with suspected cervical spondylotic myelopathy.
\end{abstract}

Traditionally patients in the UK suspected of having cervical spondylotic myelopathy are usually investigated by myelography, supplemented by axial CT. Side effects, such as headache, nausea, vomiting or dizziness, occur after myelography in up to $48 \%$ of patients ${ }^{1}$ and overnight hospital admission is considered necessary. MRI is reported to be equivalent to myelography and CT myelography in the identification of the cause of cervical myelopathy, ${ }^{23}$ and may be preferred because it is non-invasive, and does not involve exposure to ionising irradiation. A pilot study in this Institute showed $92 \%$ agreement between MRI and myelography in the level of maximum cord impingement in 57 patients with spondylotic myelopathy. ${ }^{4}$ We report a prospective study to assess the use of MRI as a replacement for myelography in the routine management of patients with suspected cervical spondylotic myelopathy.

\section{Patients and methods}

The patients studied were referred to the Neuroradiological Department with a request for myelography to investigate suspected cervical spondylotic myelopathy. To achieve consistent recruitment to the study, potential patients were independently assessed by clinical examination, and had plain radio- graphs of the cervical spine. Patients considered to have myelopathy or radiculomyelopathy with spondylotic changes on plain radiographs were included. Patients with cervical radiculopathy without myelopathy, or provisionally diagnosed to have a syrinx, tumour or multiple sclerosis were excluded, as were those who had had myelography within the last year.

Patients who fulfilled these criteria were asked to accept MRI instead of undergoing myelography. However, if circumstances prevented this being performed in the following 24 hours (for example, during routine servicing) myelography was performed. This ensured that all patients studied were thought clinically to merit myelography. After MRI a report was sent to the clinician managing the patient, who was asked (in questionnaire form) the most likely final diagnosis (degenerative, vascular, congenital, tumour, syrinx, unknown), the level(s) involved, the clinician's confidence in the diagnosis (based on a linear analogue scale of $0-100 \%$ ) and the intended management (discharge, continued review, medical, surgical, other). The clinician was also asked if further imaging was thought to be necessary, and if so, whether this was to obtain more information about the cervical region, or to exclude a structural lesion elsewhere in the spine. If further imaging was requested it was performed. With the radiological report the clinician received a second questionnaire, asking how much further imaging had contributed to the final diagnosis (definite, moderate, slight or none), about changes in the confidence in the diagnosis (if any), and the relative contribution of each investigation. Finally, the clinicians were asked to indicate their intended management of the patient.

The main end point of the study was the number of patients who could be managed using MRI as the only imaging technique, compared with the number who were judged by the clinician to require a second investigation. Additional questions concerned the changes in diagnosis, the confidence in diagnosis and the management that resulted from performing a second investigation.

MRI was performed using a Picker Vista 0.15 Tesla resistive imager, with purpose built surface coils. In all patients three different pulse sequences were used to highlight the differences between disc, osteophyte, CSF and cord (fig 1); $5 \mathrm{~mm}$ sagittal multislice balanced spin echo (SE) $700 / 32,7 \mathrm{~mm}$ sagittal slice multi-echo SE 1660/26-78-156 with increasing $\mathrm{T} 2$ weighting, and oblique axial multislice 
Figure 1 Normal examination: a) sagittal $S E 700 / 32$ b) sagittal $S E$ $1660 / 156$.
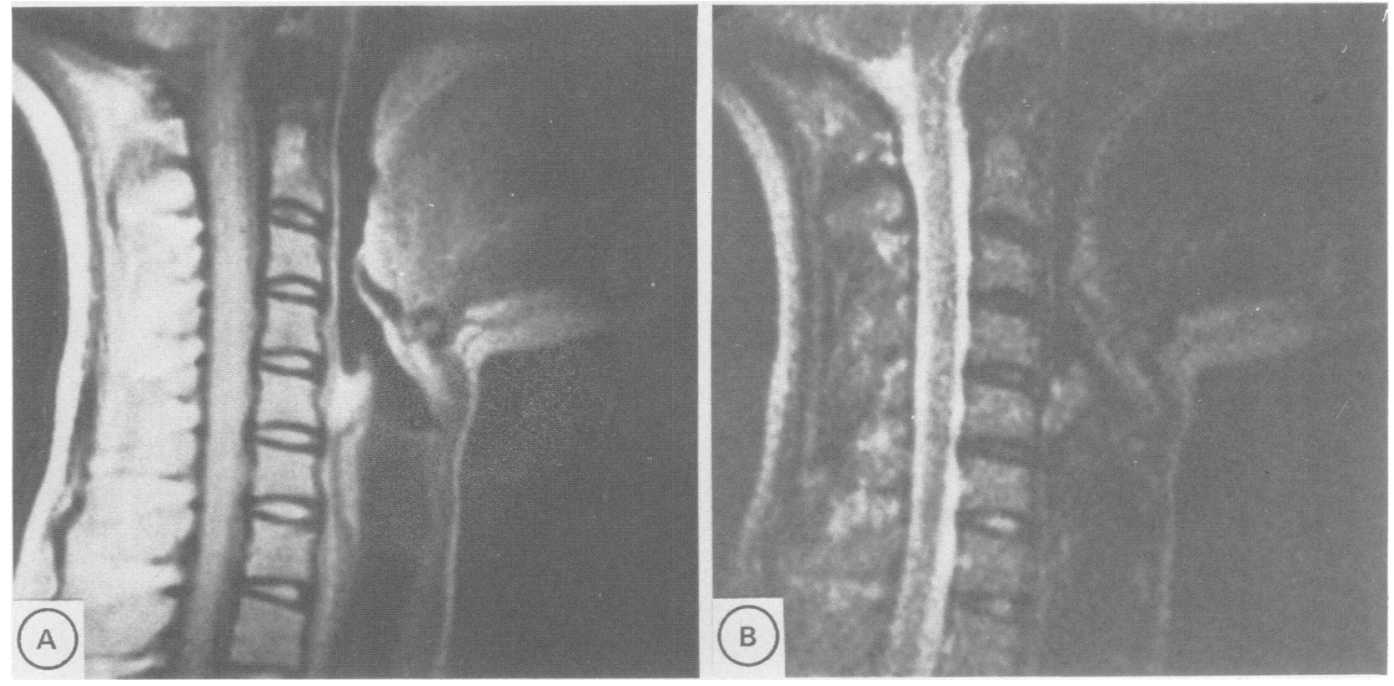

$5 \mathrm{~mm} \mathrm{~T}_{1}$ weighted SE 560/32. Myelograms were performed by running up water soluble non-ionic contrast medium introduced in the lumbar region, and CT was performed using a Phillips Tomoscan 350 with $3 \mathrm{~mm}$ axial (discangled) slices. The examinations were prospectively and independently assessed by two neuroradiologists, looking particularly at the level of maximum cord impingement and the number of levels involved. The assessment of disc impingement was based on a forced choice between normal, minimal, moderate or severe indentation of the cord. A subsidiary assessment of central or lateral (left or right) impingement was also made.

An analysis of previous myelogram requests in this department led us to expect 18 requests per month, so that within six months we could recruit over 100 patients, about a third of whom would have surgical treatment. A total of nine neurologists and six neurosurgeons were involved in the study. Approval for the study was obtained from our Institute's ethical committee, and written informed consent was obtained from each patient having MRI. The data was analysed using Chi-square tests with one degree of freedom.

\section{Results}

Patients studied

One hundred and nine patients potentially eligible for entry into the study were identified between December 1987 and August 1988. One hundred and two of these had MRI performed. Three patients were too claustrophobic and one patient had an intracranial aneurysm clip which contra-indicated MRI. Three patients were overlooked in error. The 102 patients entered were aged between 20 and 80 years, the median age being 55 years. Fifty

Table 1 Pattern of neurological abnormality

Myeloradiculopathy Myelopathy Central cord syndrome Hemicord syndrome nine were male and 43 were female. The duration of the patients symptoms ranged from one month to over five years, and in most was at least one year. The clinical features are summarised in table 1 .

During the period of the study, 396 myelograms were also performed in the same department. In 335 cases the reason for myelography was to investigate potential thoracic or lumbar disease, whilst in 34 it was to investigate cervical radiculopathy (without myelopathy).

\section{Management after $M R I$}

Eighty two patients were managed on the result of the MRI (fig 2), and a request for additional imaging was made for only 20 patients. A myelogram was performed on 18 patients and a CT myelogram on two. In 11 of these 20 patients the clinician wanted more specific information about the cervical region, but in the remaining nine, the second investigation was requested to exclude a structural lesion in the thoracic or lumbar region, which was not included in the MRI examination. Although a greater proportion of those having a second investigation had myelopathy without radicular symptoms or signs, no clinical features clearly distinguished patients who had a second investigation from those who had MRI alone.

\section{Result of further imaging}

In 18 of the 20 patients who had a second investigation there was agreement between the results of MRI and myelography or CT myelography. In two patients there was a discrepancy in the maximal level of cord compression (fig 3). Both patients had multiple level spondylosis and had surgical treatment at multiple levels. In six patients minor lateral indentation of a single nerve root was demonstrated on myelography but not MRI. The result of further imaging increased the confidence in the diagnosis (assessed by the clinician managing the patient) by a median of only $3 \%$, although in one patient it was increased by $60 \%$. Management was not changed as a result of the second investigation.

Variation between radiologists

There was agreement between the two 
Figure 2 Severe spondylosis with cord compression by prolapsed disc at $\mathrm{CV} 3 / 4$ and $4 / 5$ shown by MRI. a) sagittal SE700/32 b) sagittal $S E 1660 / 26$.
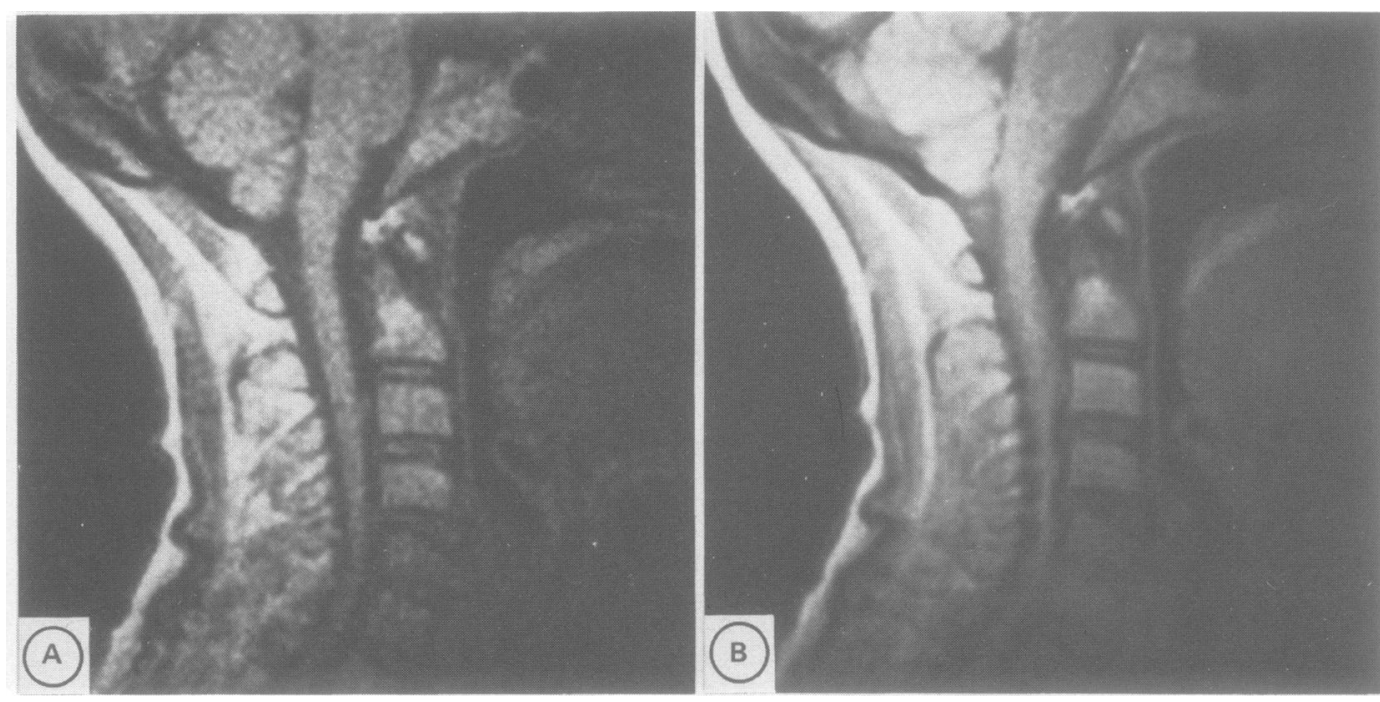

independent radiological reports in all but two patients with major cord impingement. In one patient there was a discrepancy in the degree of cord compression reported on MRI. One radiologist described severe indentation of the cord at $\mathrm{C} 5 / 6$, and the second described a large disc protrusion adjacent to, but not impinging on, an atrophic cord. The latter report was

Figure 3 Multiple level spondylosis a) myelogram, prone lateral: cord impingement at $\mathrm{CV} 4 / 5$, $5 / 6$ and 6/7, maximal at CV5/6, b) sagittal $M R I$ SE1660/156 and

corresponding $c$ ) sagittal SE700/32 showing similar cord impingement by disc and adjacent osteophytes but interpreted as maximal at $C B 6 / 7$.
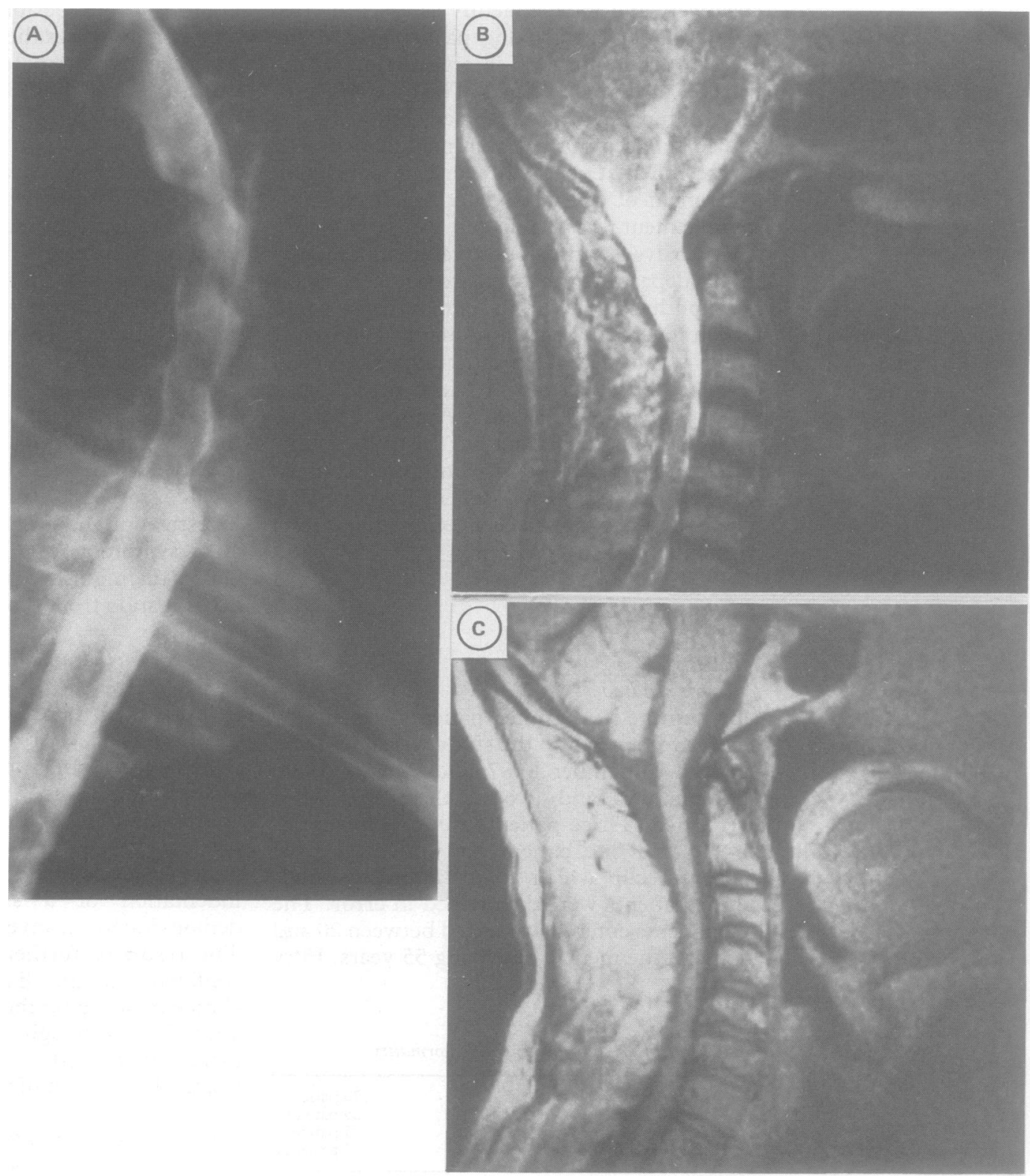
Table 2 Final diagnosis

\begin{tabular}{lll}
\hline & $\begin{array}{l}\text { Patients having } \\
\text { MRI only (82) }\end{array}$ & $\begin{array}{l}\text { Patients who had } \\
\text { additional imaging } \\
\text { after MRI (20) }\end{array}$ \\
\hline Cervical spondylotic & & \\
myelopathy & 52 & 10 \\
Demyelination & 8 & 1 \\
Tumour & 5 & 0 \\
Infection & 2 & 0 \\
Other & 8 & 2 \\
Unknown & 7 & 7 \\
\hline
\end{tabular}

*Congenital (3), rheumatoid arthritis (2), motor neuron disease (2), instability (1), post-irradiation (1), infarction (1).

Table 3 Operations performed

\begin{tabular}{lll}
\hline & $\begin{array}{l}\text { Patients having } \\
\text { MRI only }\end{array}$ & $\begin{array}{l}\text { Patients who had } \\
\text { additional imaging } \\
\text { after MRI }\end{array}$ \\
\hline $\begin{array}{l}\text { Total number of } \\
\text { patients }\end{array}$ & 82 & 20 \\
$\begin{array}{l}\text { Number who had an } \\
\text { operation }\end{array}$ & 34 & 5 \\
$\begin{array}{l}\text { Type of operation: } \\
\text { Anterior procedure }\end{array}$ & 22 & 2 \\
Posterior procedure & 8 & 2 \\
Other & 4 & 1 \\
\hline
\end{tabular}

accepted by the clinician, and he was treated without operation. In one other patient there was a discrepancy in the level of cord compression seen at myelography. One radiologist described partial obstruction to flow of contrast at $\mathrm{C6} / 7$, as well as cord indentation at $\mathrm{C} 5 / 6$. The second radiologist reported major impingement at C5/6 only, which had also been reported on MRI by both radiologists. The single level impingement was accepted by the clinician, and the patient proceeded to anterior surgery at $\mathrm{C} 5 / 6$ with good clinical effect.

Further investigations and diagnosis

In two patients the diagnosis was altered from that made after MRI as a result of subsequent investigation. In one patient the result of a test for rheumatoid factor was positive, although the myelogram was normal, and in another patient the myelogram was thought to make the diagnosis of cord infarction more likely than cord atrophy. In 15 patients a CSF sample was obtained for further diagnostic studies after MRI. In seven patients CSF was taken at the time of myelography, but eight patients needed a lumbar puncture to obtain CSF for immunological studies. This was of definite diagnostic value in only three patients.

The final diagnoses made are summarised in table 2. Almost two thirds of the patients were

Examinations making a definite contribution to diagnosis

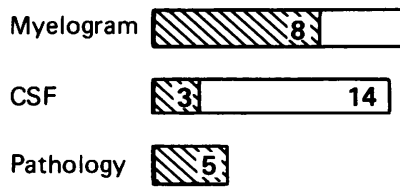

Figure 4 Investigations making a definite contribution to diagnosis. The shaded area represents the number of patients in whom the clinician felt the investigation made a definite contribution to the diagnosis, based on a forced choice between "definite", "moderate", "slight" or "no" contribution. The total area represents the total number of patients undergoing the investigation. confirmed to have cervical spondylotic myelopathy. The 40 other patients had a variety of diagnoses which included demyelination, tumours (neurofibroma in two, meningioma, astrocytoma, ependymoma), and paravertebral infections (one staphylococcal, one tuberculous). In 14 patients a diagnosis was not established. This was significantly more common in those who had a second investigation compared with those who had MRI alone ( $p<$ 0.01 ). There was no other statistically significant difference between the two groups.

\section{Operations}

Thirty nine patients had an operation after imaging (table 3 ). In each patient the expected findings were confirmed, including the five patients with tumours and the two with paravertebral infections. Of the 82 patients who had MRI alone, 34 had surgical treatment. Of the 20 patients who had a second investigation, five had an operation. In none of these had the further test changed the intended site of operation. One had rheumatoid atlanto axial disease with subaxial spondylosis. CT myelography was requested to assess the position of the odontoid. This patient subsequently had transoral excision of the odontoid followed by posterior fixation. In two other patients there was a discrepancy between the level of maximal cord compression shown by MRI and myelogram, but both had a cervical laminectomy performed from C3 to C7. The discrepancy therefore was not crucial in management. The fourth patient had predominantly radicular symptoms and signs, with relatively mild myelopathy. MRI showed cord compression at the same level as that shown by myelography. The fifth patient had marked sensory symptoms in the trunk and legs. Although MRI showed severe cord impingement at C5/6, a myelogram was performed to exclude a coincidental thoracic lesion. This confirmed C5/6 spondylosis, although there was a discrepancy in the degree of compression at C6/7, described above.

\section{Clinician's assessment}

Clinicians found MRI made a definite contribution to diagnosis in 77 of the 102 patients $(76 \%)$ whereas myelography made a definite contribu-

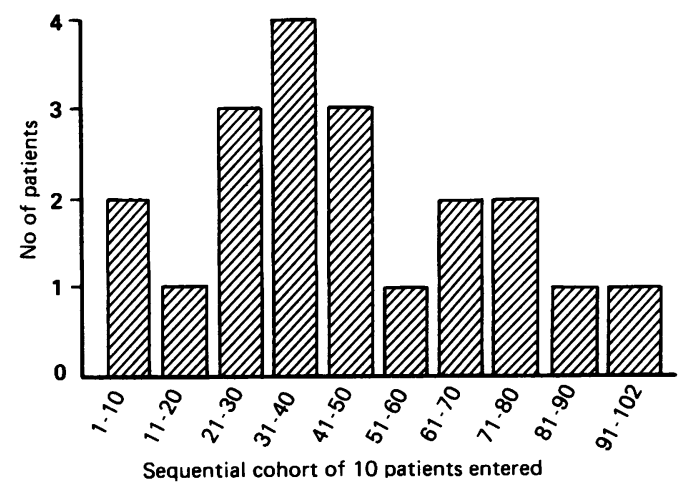

Figure 5 The influence of the study on the request for a second investigation. A comparison of the proportion of patients who had a second investigation per cohort of ten patients entered consecutively into the study. 
tion in only eight of the 20 patients $(40 \%)$ in whom it was performed after MRI. Histological examination of the lesion made a definite contribution in each of the five patients with a tumour (fig 4). Other investigations were only of definite value in a few patients; CSF studies (3), visual evoked responses (2), cranial CT (1), plain films in flexion and extension (1).

Individual clinicians entered between one and 20 patients to this study, the median number being seven. The clinician with 20 patients has a special interest in spinal surgery. Recruitment of patients to have a second investigation was not influenced significantly by the experience of previous patients in the study, with as many being recruited for a second investigation at the end of the study as at the beginning (fig 5).

\section{Discussion}

New investigative technology should be assessed as rigorously as a new surgical technique or a new drug. After initial reports that MRI of the spine produced images of high quality, comparisons of technical performance of MRI with that of conventional radiology were carried out. ${ }^{25-10}$

Studies in which patients are selected on radiological rather than clinical grounds, however, or are identified retrospectively can overestimate the value of a new test and thus misrepresent its acceptability to clinicians making management decisions. ${ }^{11}{ }^{12}$ Few studies have attempted to answer reliably the next crucial question; if MRI produces acceptable images, are they adequate for clinical management of patients with cervical spondylosis without recourse to conventional radiology?

Our results show that the use of MRI as an alternative to myelography or CT myelography facilitated accurate diagnosis and management and avoided invasive investigation in $80 \%$ of a series of consecutive patients suspected of cervical spondylitic myelopathy. Moreover, even when myelography or CT myelography was performed after MRI it did not result in a change in patient management. We did not identify a clinical subgroup of patients who would have clearly benefited from myelography as the initial investigation.

The only adverse effect of MRI reported was claustrophobia, which occurred in three patients $(2 \cdot 8 \%)$. This was more common than our experience with 600 patients having MRI of the posterior fossa among whom the incidence was $1 \% .^{13}$ Others, however, have reported this to occur in 3\% of patients having spinal MRI. ${ }^{14}$

Two thirds of the patients had a final diagnosis of spondylotic myelopathy. This is a reflection of the patient population referred in routine clinical practice. Clinically it can be difficult to establish or exclude cervical spondylosis as the cause of a myelopathy without imaging the spinal cord. An appreciable proportion of patients referred for investigation prove not to have cervical spondylotic myelopathy requiring operation.

The use of surface coils in MRI improves the signal to noise ratio, but limits the size of the region that can be examined in one scan. This was a disadvantage in patients who did not have clinical or plain radiographic features that localised the cervical spine as the site of the patients' disorder. In nine patients this left the diagnosis sufficiently uncertain after MRI that the clinician requested a myelogram to exclude a thoracic or lumbar structural lesion. Each of these investigations was normal below the cervical cord.

The design of our study meant that the patients were investigated as an "in patient", so that myelography could be done if MRI was not available. If this had not been the case, MRI could have been performed as an outpatient. Then, only those needing a lumbar puncture for CSF studies would have required overnight hospital admission. Using our pulse sequences at $0 \cdot 15$ Tesla, MRI of the cervical cord takes about one hour to perform, significantly longer than a run-up myelogram. However, the current use of more stable higher magnetic field strength imagers reduces the time needed to obtain an image, because the signal to noise ratio is higher, which necessitates fewer "averages" of information to produce the same quality of image. In addition, thinner slices $(<5 \mathrm{~mm}$ ), 3D volume acquisitions, and low flip angle sequences can be obtained, resulting in faster imaging and better image quality..$^{15-17}$

The resource implications of a change to the use of MRI for investigation of cervical spondylotic myelopathy are being assessed in parallel with this study. The factors to be considered include, in addition to capital and running costs, the benefit of investigation as an outpatient. Although an allowance must be made for patients who may subsequently need overnight hospital stay for a myelogram or lumbar puncture, these will have a low diagnostic yield. Overall it may be possible to release appreciable in patient facilities for other uses. On the other hand, the greater ease of investigation as an outpatient may lead to an increase in the number of patients being referred, and an increase in total costs.

The decision to investigate a patient suspected of having cervical spondylotic myelopathy is based on the perceived balance of risks; investigation (at present myelography) and surgical treatment, versus benefits; the establishment of a diagnosis and institution of appropriate management. Our study shows that clinicians found MRI a satisfactory alternative to myelography, providing sufficient information for appropriate management in $80 \%$ of cases. With routine use of MRI the risk/benefit balance may favour earlier investigation, and this may lead to more successful treatment.

This study was financed by an MRC research grant $1 \mathrm{D} 81$. We thank Audrey Lawrence for statistical advice, all the clinician who contributed their time to this study, and Ann Semple for typing the manuscript. 
1 Macpherson P, Teasdale E, Coutinho C, McGeorge A. Iohexol versus Iopamidol for cervical myelography: a randomised double blind study. $\mathrm{Br} J$ Radiol 1985;58: 849-51.

2 Masaryk TJ, Modic MT, Geisinger MA, et al. Cervical myelopathy: a comparison of magnetic resonance and myelopathy: a comparison of magnetic

3 Modic MT. Degenerative Diseases of the spine. In: Modic MT, Masaryk TJ, Ross JS, eds. Magnetic resonance imaging of the spine. London: Year Book Medical Publishing, 1989:75-119.

4 Hadley D, Macpherson P. MRI can replace myelography in the investigation of degenerative cervical myelopathy. $B$ J Radiol 1988;61 (Abst):750.

5 Modic MT, Weinstein MA, Pavlicek W, et al. Magnetic resonance imaging of the cervical spine. Technical and clinical observations. Am J Neuroradiol 1984;5:15-22.

6 Hymen RA, Edwards JH, Vacirca SJ, Stein HL. 0.6T MR Imaging of the Cervical Spine: Multislice and Multiecho techniques. Am J Neuroradiol 1985;6:229-36.

7 Modic MT, Masaryk TJ, Ross JS, Mulopulos GP, Bundschuh CV, Bohlman $\mathrm{H}$. Cervical radiculopathy: value of oblique MR imaging. Radiology 1987;163: value of

8 Karnaze MG, Gado MH, Sartov KJ, Hodges FJ. Comparison of MR and CT myelogram in imaging the cervical and thoracic spine. Am J Neuroradiol 1987;8:983-9.

9 Brown B, Schwartz RH, Frank E, Blank NK. Preoperative Evaluation of Cervical Radiculopathy and Myelopathy by surface coil MR Imaging. Am J Neuroradiol 1988;9:
$859-66$.

10 Larsson EM, Holtas S, Cronquist S, Brandt L. Comparison of myelography, CT myelography and magnetic resonance imaging in cervical spondylosis and disc herniation. Pre and post operative findings. Acta Radiologica 1989;30: 233-9.

11 Kent DL, Larson EB. Diagnostic technology assessments: problems and prospects. Ann Int Med 1988;108;5:759-61.

12 Cooper LS, Chalmers TC, McCally M, Berrier J, Sacks HS The poor quality of early evaluations of magnetic resoThe poor quality of early evaluations of magnetic reso-

13 Teasdale GM, Hadley DM, Lawrence A, et al. Computed tomography or magnetic resonance imaging for a possible posterior cranial fossa lesion? A randomised prospective study. $B M J$ 1989;299:349-55.

14 Modic MT, Masaryk TJ, Mulopulos GP, Bundschuh C Han J, Bohlman H. Cervical radiculopathy: prospective evaluation with surface coil MR imaging, CT with metrizamide, and metrizamide myelography. Radiology 1986;161:753-9.

15 Enzman DR, Rubin JB, Wright A. Cervical spine MR imaging: generating high signal CSF in sagittal and axial images. Radiology 1987;163:233-8.

16 Hedbers MC, Drayer BP, Flom RA, Hodak JA, Bird CR. Gradient echo (GRASS) MR imaging in cervical radicuGradient echo (GRASS) MR imaging in cer

17 Enzman DR, Rubin JB. Cervical spine: MR imaging with a partial flip angle, gradient refocussed pulse sequence. Pt 1 . partial flip angle, gradient refocussed pulse sequence. Pt 1 ; 166:467-72. 